\title{
Social determinants and mortality
}
OP50 SOCIO-DEMOGRAPHIC DIVERSITY AND UNEXPLAINED VARIATION IN DEATH RATES BETWEEN THE MOST DEPRIVED AREAS IN BRITAIN

H Tunstall, ${ }^{1 *}$ R Mitchell, ${ }^{2}$ D Dorling, ${ }^{3} \mathrm{~J} \mathrm{Gibbs}^{4}{ }^{4} \mathrm{~S}$ Platt ${ }^{5}{ }^{1}$ Health Sciences, University of York, York, UK; ${ }^{2}$ Public Health, University of Glasgow, Glasgow, UK; ${ }^{3}$ Geography, University of Sheffield, Sheffield, UK; ${ }^{4}$ Freelance Researcher and Consultant, Montreal, Canada; ${ }^{5}$ Public Health Sciences, University of Edinburgh, Edinburgh, UK

10.1136/jech.2011.143586.50

Background There is a strong relationship between area death rates and deprivation in Britain. However, there is also considerable unexplained variation in death rates between the most deprived cities and regions.

Objectives To explore heterogeneity in death rates among deprived areas in Britain by assessing the degree of variation in death rates and socio-demographic factors among area deprivation deciles and how the variables associated with death rates differ between the most deprived decile and areas with all levels of deprivation.

Methods Age- and sex-standardised death rates 1996-2001, Carstairs 2001 score, proportion of Carstairs deprivation indicators (male unemployment, no car, low social class and overcrowding) and black and minority ethnic group (BME), population density and population change 1971-2001 were calculated for 641 parliamentary constituencies in Britain. Equal numbers of constituencies were grouped into Carstairs deciles. Standard errors were calculated for all variables by deprivation decile. The associations between death rates and the socio-demographic variables were assessed with Pearson's correlations by decile and for all constituencies. Multivariate relationships between the dependent variable, death rates, 
and the socio-demographic variables were explored in linear regression models.

Results Standard errors for death rates and most sociodemographic variables were greatest for the most deprived decile. Death rates among all constituencies were positively correlated with Carstairs, the deprivation indicators, population density and BME but for the most deprived decile no association was found with Carstairs and there was a negative correlation with overcrowding, population density and BME. Population change was the variable most strongly correlated with deaths in the most deprived decile and was negatively associated among this group and all constituencies. In multivariate analysis of the most deprived decile models containing population density, BME and change had substantially higher $\mathrm{R}$ Squares than those containing only Carstairs deprivation indicators. In analysis of all socio-demographic variables in models for the most deprived decile and all constituencies the direction of association between death rates and overcrowding, population density and BME was the same and the strongest associations were with no car and population density.

Conclusion There is greater variation in death rates and socio-demographic characteristics among the most deprived constituencies. The socio-demographic factors that are most strongly correlated with death rates among the most deprived places differ from areas of all deprivation levels and include population density, ethnicity and migration. Explanations of variations in death rates between deprived areas may be supported by better understanding of their socio-demographic heterogeneity. 